Journal of Antimucrobial Chemotherapy (1985) 15, Suppl A, 201-206

\title{
In-vivo assessment of in-vitro killing patterns of Pseudomonas aeruginosa
}

\author{
A. U. Gerber and C. Feller-Segessenmann \\ Department of Medicine, Inselspital, and Institute of Medical Microbiology, \\ University of Bern, Switzerland
}

\begin{abstract}
Time-kıll curves of Pseudomonas aeruginosa exposed to gentamicin or ticarcillın in vitro were correlated with time-kill curves obtained with various dosage schedules of the same study drugs in granulocytopenic mice. An instantaneous, fast and drugdependent killing pattern was found in vitro with gentamicin. This pattern corresponded to bacterial killing in vivo which was clearly dependent on peak drug levels. In contrast, slow bacterial killnng with little relationship to concentration was found in vitro with tucarcillin and proved to correlate with an antibacternal effect in vivo seen at trough levels. We conclude that in-vitro time-kill curves of antımicrobial agents may be predictive for optimizing dosage regimens in vivo.
\end{abstract}

\section{Introduction}

The in-vitro activity of an antibiotic can be well established in time-kill curves as obtained by exposure of the test bacteria to various concentrations of drug. In similar experiments, the regrowth pattern of bacteria can be studied in vitro after short exposure of the organisms to the study drug. Using such technique, Bundtzen et al., (1981) and Gerber \& Craig (1981) have shown that exposure of various Gram-negative bacteria to inhibitors of protein- or nucleic acid-synthesis is followed by a persistent post-antibiotic effect (PAE); i.e. a persistent suppression of bacterial growth upon removal of the drug. In contrast, virtually no PAE was found after exposure of Gramnegative rods to $\beta$-lactam drugs.

Little is known about how killing and regrowth patterns observed in vitro relate to antimicrobial activity of antibiotics in vivo. In the present study, therefore, experiments were devised to establish time-kill curves of the same pairs of drug and organism not only in vitro but also in vivo. A hypothesis was proposed that in-vivo efficacy of an antimicrobial agent is related to peak plasma levels whenever a fast bactericidal drug effect and a PAE are found in vitro, whereas slow bacterial killing and absence of a PAE in vitro indicate that in-vivo antimicrobial activity depends not on peak- but mainly on trough drug levels.

\section{Materials and methods}

Pseudomonas aeruginosa ATCC 27853 was the main study organism. Two clinical isolates of Ps.aeruginosa were included in confirmatory experiments. Ticarcillin

This work was supported by the Swiss National Foundation for Scientufic Research, Grant no. $3865-081$

Reprint requests to Dr Andreas U Gerber, Department of Medicine, University of Bern, Inselspital, CH-3010 Bern, Switzerland 
(Beecham) and gentamicin (Shering, U.S.A.) were the drugs used. In-vitro time-kill curves were constructed after exposure of the organisms to various concentrations of drug in Mueller-Hinton broth supplemented with $\mathrm{Ca}^{++}$and $\mathrm{Mg}^{++}$according to Stratton \& Reller (1977). The number of colony-forming units (cfu) was determined by plating serial dilutions of appropriate culture samples onto tryptic soy agar plates. Methods for in-vivo studies have been described by Gerber, Craig \& Brugger (1983). Agranulocytic (cyclophosphamide treated) mice were used. On the day of the experiment, mice were injected with $10^{7} \mathrm{cfu}$ of the organisms in the thigh muscle. Two hours later treatment was started with sc injections of the study drug. In some experiments mice were randomized and treated with either a $1-\mathrm{h}$ or a $3-\mathrm{h}$ regimen such that identical total amounts of injected drug resulted in both groups after 3,6,9 and $12 \mathrm{~h}$ of treatment. Blood was drawn from the retro-orbital sinus of all mice, and plasma drug levels were determined using a biological method (Bacillus subtilis as indicator organism). Antimicrobial activity was assessed by viable counts in homogenized thighs of killed animals, and time-kill curves were constructed.

\section{Results}

Killing patterns of $\mathrm{Ps}$. aeruginosa in vitro

Time-kill curves of Ps.aeruginosa ATCC 27853 obtained in vitro with various concentrations of gentamicin and ticarcillin are shown in Figure 1. A substantial difference between the two drugs was found regarding the time course of the bactericidal effect. Killing by gentamicin was fast, very much concentration-dependent, but followed by bacterial break-through growth. On the other hand, ticarcillin needed

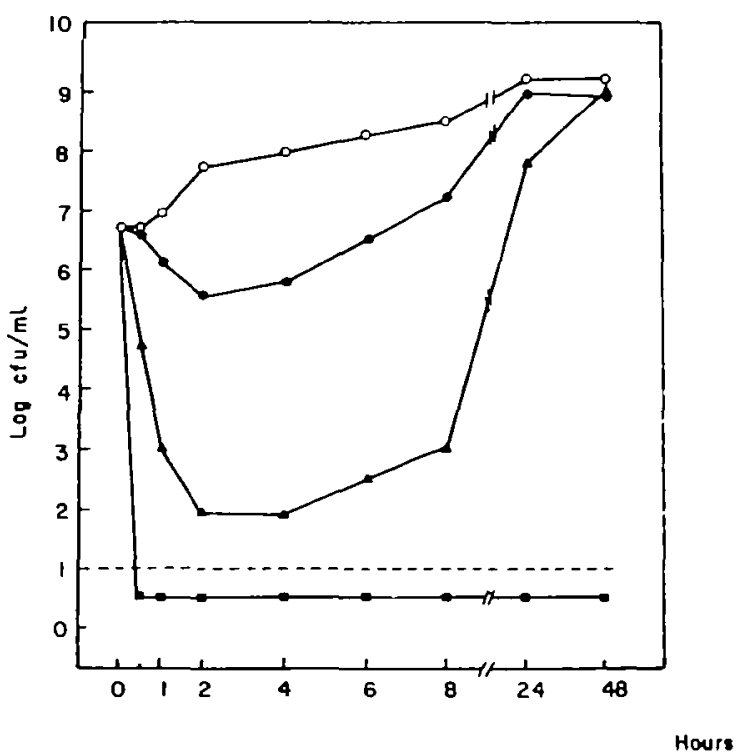

(a)

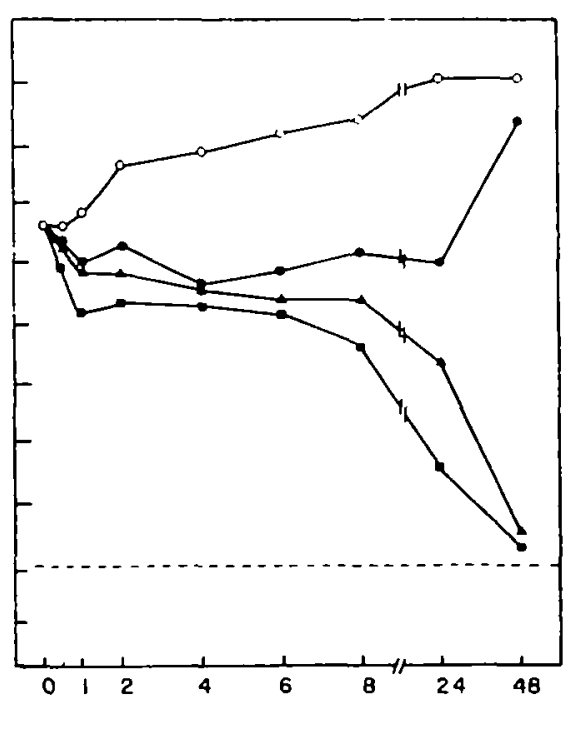

(b)

Figure 1. In-vitro time course of the bactericidal effects of gentamian (a) and tucarcallun (b) on Ps. aeruginosa ATCC 27853 in calcium- and magnesium-supplemented MHB. Standard MICs (MBCs) for $6 \times 10^{5}$ organisms were $2(8)$ and $32(32) \mathrm{mg} / 1$ of gentamicin and ticarcallin, respectively $\mathrm{O}$, Control; $\bullet$,
half-fold MIC; $\triangle$, two-fold MIC, $\square$, ten-fold MIC. 
several hours to exert a $99 \%$ bactericidal effect which was poorly concentrationdependent No break-through growth was observed at levels greater than the MICs of ticarcillin.

\section{Comparative killing patterns in vivo}

Killing patterns similar to those obtained in vitro were observed with high dose treatment of Ps.aeruginosa ATCC 27853 in the thigh infection model using granulocytopenic mice (Figure 2). Gentamicin resulted in $>90 \%$ bacterial killing in the first $6 \mathrm{~h}$ of treatment, but a significant break-through growth was observed thereafter, although treatment continued and plasma levels far above the MIC were obtained, at least intermittently. The bactericidal effect of ticarcillin on the other hand was slow and bacterial break-through growth did not occur up to $24 \mathrm{~h}$ of high dose treatment.

Additional experiments were performed with lower doses of drug to see the effect of dosage schedule on antipseudomonal activity in vivo (Figures 3 and 4).

\section{Importance of dosage schedules of gentamicin}

Experiments with gentamicin (Figure 3) clearly revealed the key importance of peak levels for the antimicrobial effect of this drug. By $3 \mathrm{~h}$ of treatment, a single injection of $15 \mathrm{mg} / \mathrm{kg}$ of gentamicin (resulting in peak plasma levels of approximately $20 \mathrm{mg} / \mathrm{l}$ ) was clearly more effective than the same total amount of drug subdivided in three fractional doses injected every hour and resulting in peak plasma levels of
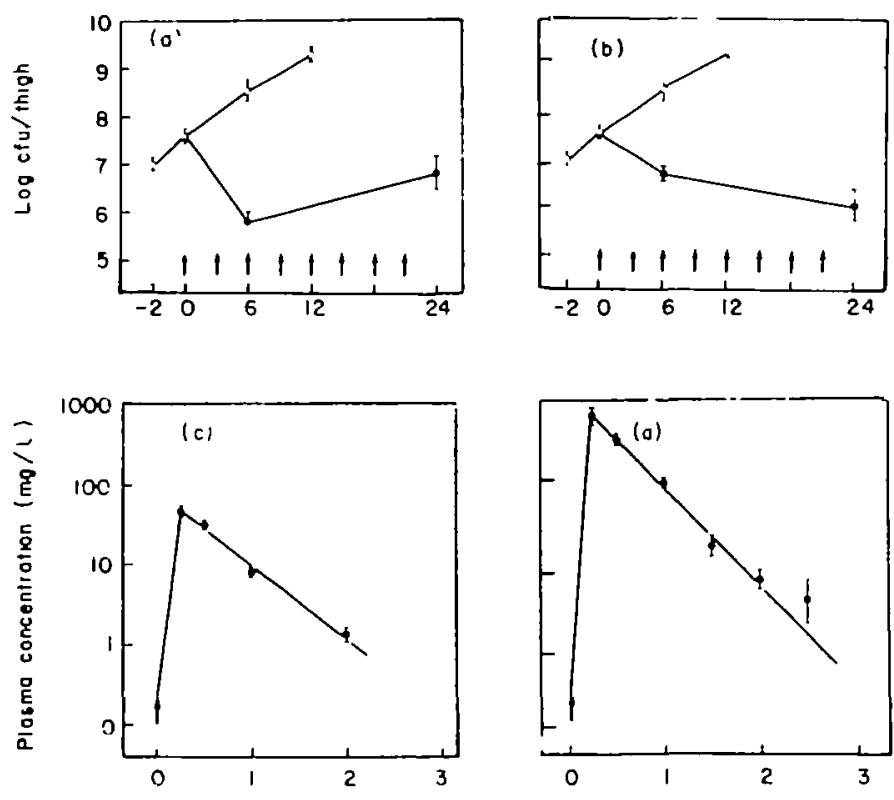

Hours

Figure 2 Comparatıve in-vioo kslling patterns of a 3-h schedule of gentamıcin (a), and tucarcallin (b), on Ps aeruginasa ATCC 27853 (granulocytopente mice) The plasma kinetucs of a single dose of gentamicin and ucarcalin are shown on a loganthmic scale in the (c) and (d) Each point represents the mean value \pm s.D of three mice See Figure I for MIC and MBC-values 


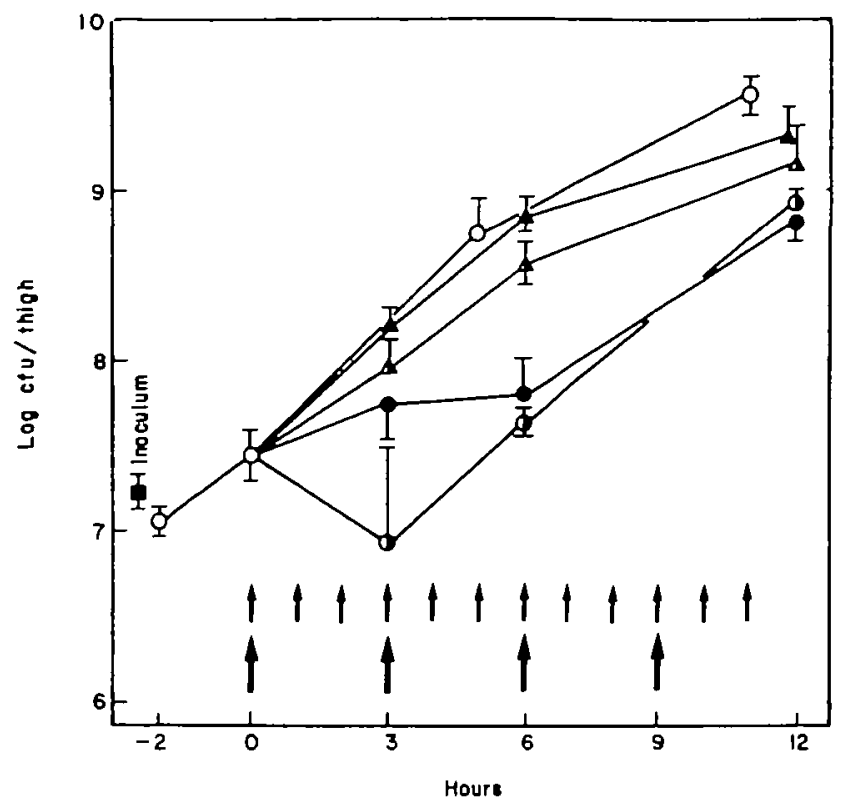

Figure 3. Importance of dosage schedules of gentamicin on Ps aeruginosa ATCC 27853 in vivo (granulocytopenic mice)

The effects of two 1-h regimens are compared to two 3-h regimens. Identical total amounts of drug had been injected in comparatively treated mice after $3,6,9$, and $12 \mathrm{~h}$. O, Control; $\Delta, 1.67 \mathrm{mg} / \mathrm{kg} \mathrm{q} 1 \mathrm{~h} ; \Delta$, $5 \mathrm{mg} / \mathrm{kg} \mathrm{q} 3 \mathrm{~h}, 0,5 \mathrm{mg} / \mathrm{kg}$ q $1 \mathrm{~h}, 0,15 \mathrm{mg} / \mathrm{kg} \mathrm{q} 3 \mathrm{~h}$.

approximately $6-8 \mathrm{mg} / \mathrm{l}$. Break-through growth could not be prevented by either of the two gentamicin dosage regimens.

\section{Importance of dosage schedules of ticarcillin}

The time course of the antipseudomonal effect of ticarcillin and the impact of dosage intervals of this drug were studied in granulocytopenic mice infected simultaneously with two clinical isolates of $P$ s. aeruginosa which differed substantially in virulence and susceptibility to ticarcillin (Figure 4). In contrast to strain E 29/2, strain 14974 was unable to grow in the thigh muscle of non-granulocytopenic mice. In vitro as well as in granulocytopenic mice strain 14974 multiplied slower than strain E 29/2. The colonies formed by the two strains on agar plates (small for strain 14974, larger and mucoid for strain E 29/2) could easily be distinguished.

Identical inocula of the two strains were injected into left and right thighs, respectively, of the same mice. Treatment was started $2 \mathrm{~h}$ later at a $1 \mathrm{~h}$ vs. a $3 \mathrm{~h}$ schedule, using identical total amounts of drug in both groups of mice treated. Against both Ps. aeruginosa strains tested the only effect of the $3 \mathrm{~h}$ regimen was to slow down bacterial growth. However, a complete bacteriostatic or bactericidal effect was not obtained, although peak plasma levels exceeded by at least two-fold the MIC for strain E 29/2 and by 64 -fold the MIC for strain 14974. One hour injections of fractional doses of the same total amount of ticarcillin proved to be significantly more efficaceous against both strains tested despite three-fold lower peak plasma levels which, for strain E 29/2, did not even reach the MIC. Thus, the activity of ticarcillin was mainly 

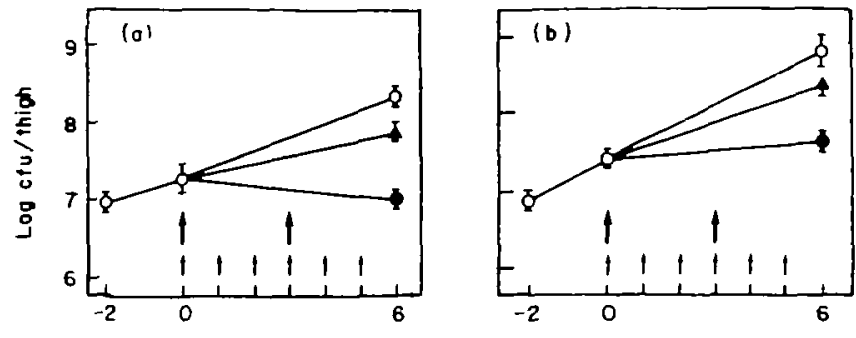

Hours

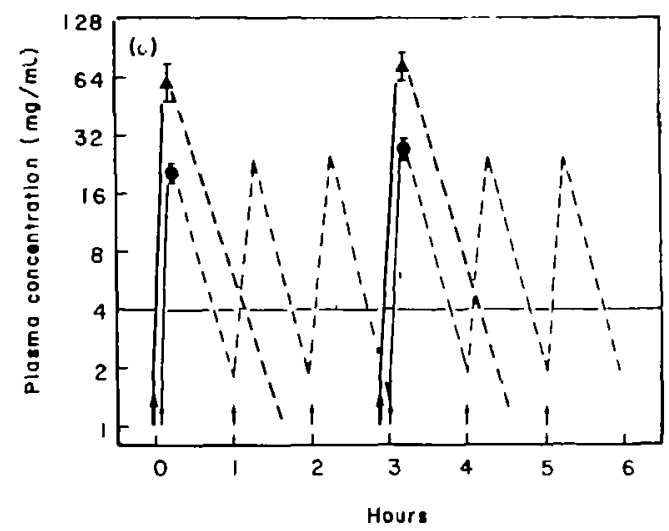

Figure 4. Importance of dosage schedules of tucarcillun on actuvity against two clinical isolates of Ps aeruginosa (a) 14974, MIC $1 \mathrm{mg} / \mathrm{l}$; (b) E 29/2, MIC $32 \mathrm{mg} / \mathrm{l}$ Peak plasma levels of tucarcillin and presumptuve kinetics of the two dosage regumens is shown in (c). $O$, Control; $\Delta, 90 \mathrm{mg} / \mathrm{kg} \mathrm{q} 3 \mathrm{~h} ; 0,30 \mathrm{mg} / \mathbf{k g}$ i h Four $\mathrm{mg} / \mathrm{l}$ of ticarcallin was the lower limit of detectability in the biological assay used Identical total amounts of drug had been injected in both regimens by 3 and $6 \mathrm{~h}$ of treatment

dependent on constant presence of the drug rather than on peak levels far in excess of the MIC.

\section{Discussion}

Our experiments clearly demonstrate the difference between gentamicin and ticarcillin regarding the time course of their pharmacological response in vitro as well as in vivo. In vivo the antipseudomonal activity of gentamicin proved to be dependent on peak plasma levels. This finding reflected the dose-response curve observed with gentamicin in vitro which showed that increasing concentrations of gentamicin increased both absolute bacterial killing and the speed of killing. In addition, Bundtzen et al. (1981) have demonstrated a PAE of gentamicin on Ps. aeruginosa which lasted 1.6-2.6 h. This effect may thus cover, at least in part, the period of sub-MIC plasma levels between doses and prevent early bacterial regrowth during that phase of treatment. In conclusion, a gentamicin-type response, i.e. peak-dependent killing and a PAE, may well indicate an advantage in vivo of high dose, long interval treatment over a low dose, short interval regimen of any antimicrobial agent. This interpretation of the present data is in full agreement with our previous work (Gerber et al. 1983) and with the results of recent investigations by Powell, Thompson \& Luthe (1983) who found once daily doses of aminoglycosides to be more effective than more frequent injections. 
Interestingly, in those studies once daily dosing of aminoglycosides was also less toxic than the conventional $8 \mathrm{~h}$ schedule.

Our in-vivo results with ticarcillin were not surprising. Bacterial killing in vitro was slow and showed a poor correlation with concentration. High peak levels greatly exceeding the MIC seem to be of little value in vivo. On the other hand, the half-life of ticarcillin in mice was shorter than $20 \mathrm{~min}$. It is therefore possible that the action time was just too short to exert any bactericidal effect. Since the half-life in man is considerably longer than in mice, a drug showing a response similar to that of ticarcillin might be underestimated when investigated in mice (Gerber et al., 1983). However, it should be remembered that ticarcillin clearly lacks a PAE on Ps. aeruginosa in vitro (Bundtzen et al., 1981). Therefore, it is logical to conclude that any antibiotic showing a response like that of ticarcillin does require super-MIC drug levels at all times in order to exert its maximal antimicrobial activity in vivo. This activity must therefore be associated with trough plasma levels.

\section{References}

Bundtzen, R. W., Gerber, A. U., Cohn, D. L. \& Craig, W. A. (1981). Postantibiotic suppression of bacteral growth. Reviews of Infectious Diseases 3, 432-8.

Gerber, A. U. \& Craig, W. A. (1981). Growth kinetics of respiratory pathogens after short exposures to ampicillin and erythromycin in vitro. Journal of Antimicrobial Chemotherapy 8 , Suppl. C, 81-91.

Gerber, A. U., Brugger, H. P., Stritzko, T., Stalder, B. \& Feller, C. (1983). Antipseudomonal activity of ceftazidime, ceftriaxone and ticarcillin in leukopenic mice. Impact of human versus murne pharmacokinetics. 23rd Interscience Conference on Antimicrobial Agents and Chemotherapy, Abstract No. 363.

Gerber, A. U., Craig, W. A., Brugger, H. P. et al. (1983). Impact of dosing intervals on activity of gentamicin and ticarcillin against Pseudomonas aeruginosa in granulocytopenic mice. Journal of Infectious Diseases 147, 910-7.

Powell, S. H., Thompson, W. L., Luthe, M. A. et al (1983). Once-daily vs. contunuous aminoglycoside dosing. Efficacy and toxicity in animal and clinical studies of gentamicin, netilmicin, and tobramycin. Journal of Infectious Diseases 147, 918-32.

Stratton, C. W. \& Reller, L. B. (1977). Serum dilution test for bactencidal activity. I. Selection of a physiologic diluent. Journal of Infectious Diseases 136, 187-95 